\title{
COSTA Y SIERRA EN EL ANTIGUO PERU
}

\author{
WeNDELl C. BeNNETT
}

Prácticamente todos los arqueólogos consideran la prehistoria peruana como un todo unificado. Esto es más que una conveniencia de identificación, desde que los restos arqueológicos de la Costa y la Sierra del Perú están suficientemente relacionados como para formar un segmento integrado de historia cultural. En realidad, algunos investigadores describen el antiguo Perú como un área cultural con profundidad en el tiempo, por el cual se ha propuesto el término "área de cotradición (Bennett, 1948).

Al tiempo de la conquista española, los Incas ya habían unificado esta área, además de una considerable extensión de áreas vecinas, dentro de su imperio políticamente unificado. Las fronteras políticas tienen a menudo poco que hacer con la unidad cultural, pero en este caso las culturas componentes della parte peruana del imperio compartían suficientes características como para que esté justificado el término de área cultural. Parece que una unidad similar aparece por lo menos 2,000 años antes de la expansión Inca, aunque no haya evidencia alguna de un control político pan-peruano en períodos anteriores.

Es posible describir las características principales de la "co-tradición peruana" y examinar sus rasgos de desarrollo a través del tiempo y del espacio. Además, uno puede comparar este segmento de historia cultural con otros centros, tales como Meso-América y el Sudoeste de Estados Unidos. En realidad estas comparaciones más amplias conducen la unidad de la prehistoria peruana hacia un foco más delimitado.

\section{SUBDIVISIONES}

Aunque los investigadores están en acuerdo general sobre la unidad fundamental del pasado peruano, todos reconocen subdivisiones de esta unidad, tanto espaciales como temporales. Algunos subdividen el Perú segmentos geográficos dentro de los que se desarrollaron culturas locales con rasgos y tradiciones características. Otros, como el finado, y notable doctor Tello, imaginan el crecimiento cultural igual al 
de un árbol, con un tronco central y raíces y ramas extendidas. Este modo de ver las cosas recalca las relaciones de la unidad cultural donde quiera que se encuentren geográficamente. Ambas formas son defendibles y difieren únicamente $\in n$ los rasgos que remarcan.

Aquellos que tratan de las subdivisiones geográficas aislan generalmente grupos de valles costeños, tales como Chicama - Moche Virú en la costa norte y las cuencas serranas básicas, tales como las del Titicaca, Mantaro y Cajamarca. Inevitablemente la sierra se distingue de la costa. Esto refleja la magnitud de los contrastes entre esos ambientes que han impresionado a geógrafos, historiadores, economistas y novelistas, así como a los arqueólogos. Ciertamente es difícil ignorar las saltantes diferencias entre la cordillera yerma, sin árboles, rodeada de majestuosos picos nevados y las llanuras de los desiertos costeños cortadas por tortuosos ríos y cuyos valles son fértiles y verdes sólo cuando se controla la irrigación. En realidad, algunos estudiosos, como Means (1931) llegaron a describir la cultura básica de la sierra como opuesta a aquella de tipo costeño.

Evidentemente existen muchas diferencias culturales entre la costa y la sierra: las construcciones de piedra son comunes en la sierra, raras en la costa; la cría de la llama estuvo restringida a las alturas, los sembríos de algodón a los llanos. No obstante, si la prehistoria peruana forma una unidad debe incluir tanto a las regiones de la sierra como de la costa. Por tanto, parece apropiado examinar los tipos de relaciones entre estas dos divisiones principales. Esto a su vez ofrecerá una base para examinar la magnitud de diferencias culturales, que tal vez se hayan exagerado un poco, según se deduce por Rowe (1948, p. 20): "Mi impresión del cuadro arqueológico peruano es que las diferencias entre las sub-áreas de la sierra y las sub-áreas de la costa son apenas mayores de lo que uno puede encontrar en subláreas"serranas vecinas. Hasta en términos geográficos las diferencias entre las sub-áreas de la sierra 'son muy grandes".

Antes de volvernos hacia el problema de las relaciones deben sin embargo considerarse algunos de los factores que pueden haber afectado el desarrollo cultural en la costa y en la sierra, aunque sea en forma muy esquemática.

\section{LA COSTA VS. LA SIERRA}

1. Habitabilidad. - Las únicas áreas habitables de la desierta faja costera son los valles de los ríos que ia cortan yendo hacia el Pacífico. Fxisten unos veinticinco ríos principales, que varían considerableguiente, tanto por extensiones desérticas ellos está muy aislado del sinas. Äígunos ríos son permanentes, otros como por estribaciones andifavorablemente grandes llanuras, otros esporádicos, algunos cortan sos la habitación está limitada definidamente a las tierras que pueden irrigarse. Esto a su vez depende de las fuentes de agua, que en la mayoría de los casos está restringida a una sola. 
El aislamiento de los valles costeños, además de su dependencia de una sola fuente de agua, sugeriría que cada uno forma una unidad cultural independiente. En realidad esto parece salir de la evidencia arqueológica, que muestra las características de cada valle y además, en el caso de Virú, que la cultura fué uniforme en todo el valle en cualquier período dado de tiempo. Esto ha sido también confirmado por la revisión de Newman (1948) de la evidencia física, que muestra que la mayor parte de la gente costeña pertenecía a un tipo racial en los períodos más antiguos, que cada valle parece haber tenido un discreto centro de reproducción.

La difusión de la cultura de un valle a otro, que permite asociarse a varios como una unidad, refleja la extensión de las barreras entre ellos. Por ejemplo, el amplio desierto marca divisiones culturales entre Piura y Lambayeque, Pacasmayo y Chicama, Virú y Santa, Casma y Huarmey.

En la sierra, hay innumerables pequeñas bolsas a lo largo de muchos ríos con pequeñas agrupaciones, mas los terrenos para una habitación extensiva se encuentran únicamente en las hoyas interandinas y estructurales, de las que hay seis importantes: Cajamarca, Callejón de Huaylas, Huánuco, Mantaro, Cuzco y Titicaca. Aunque algunas de estas hoyas se caracterizan por ríos importantes, tales como el Santa y el Mantaro, no han sido formadas por los ríos, sino más bien son hoyas geológicas estructurales, tales como el extenso altiplano que contiene el Lago Titicaca. La construcción interandina de estas hoyas ofrece abundancia de terreno fértil, aunque esto esté lejos de ser uniforme, y algunos lugares hayan sido malamente erosionados. Sin embargo estas hoyas pueden soportar grandes poblaciones, que dependen de la agricultura, la que complementan con el pastoreo en las punas circundantes. Opuestamente a los valles costeños la agricultura en las hoyas no depende de una sola fuente de agua y aunque las hoyas principales están ampliamente separadas, se encuentran pequeñas agrupaciones en todos los lados. En consecuencia, la unidad cultural no está implícita para las hoyas de la sierra y ciertamente un examen último de la arqueología de la hoya del Titicaca (Bennett, 1950) muestra que nunca estuvo culturalmente unida en toda su historia, no obstante el hecho favorable del lago, que permite la fácil comunicación.

2. Altura.- Los llanos de los valles costeños tienen poca altura. Los ríos se originan en la cordillera y sus cursos altos están confinados a estrechas gargantas donde la habitación casi no es posible o lo es en muy poco grado. Sin embargo, las principales hoyas de la sierra están a gran altura, que van desde los 2,500 hasta los 4,000 metros. Los efectos culturales de la vida en la altura deben tenerse en cuenta. Las alturas elevadas restringen además de las plantas y animales que pueden ser utilizados, la energía humana. La investigación de la adaptación del hombre a grandes alturas que actualmente llevan a cabo Monge (1948) y sus colegas es de la mayor importancia. Si las necesidades de la adaptación biológica a las grandes alturas son suficientes para restringir la libre migración de las gentes de la costa a la sierra, esto 
evidentemente debe tenerse muy en cuenta en la interpretación arqueológica.

3. Clima.- La costa del-Perú, aunque situada en la zona tropical, carece de lluvias y su temperatura moderada durante el año se debe a los efectos de la Corriente Peruana a lo largo de la costa del Pacífico. En consecuencia no se requería casas o ropas que protegieran contra temperaturas extremas ni de grandes precipitaciones. Además, las construcciones de adobes secados al sol podían servir como estructuras relativamente permanentes. Sin duda que las raras lluvias fuertes causaron verdaderas destrucciones según puede observarse en el pasado arqueológico, así como en tiempos históricos. Arquelógicamente los tipos de habitación reflejan esta situación. Las construcciones tienen habitaciones pequeñas, aparentemente sólo con el propósito de servir de dormitorio, ya que las pruebas de fogones interiores son escasas. Otras pruebas muestran casas con techos de paja bordadas, frentes abiertos, y aun construcciones de esteras. Las muestras preservadas de telas tejidas muestran que el vestido era ligero y que se hacía poco uso de frazadas y otros materiales de abrigo.

En la sierra hay estaciones lluviosas con abundantes precipitaciones y las temperaturas están gobernadas por la altura, de modo que la mayoría de las áreas habitables son templadas y frías durante el año. Ropa y casa adecuada son importantes. El adobe puede usarse para construcciones, aunque las construcciones permanentes deben hacerse de piedra. Las casas arqueológicas tienen pequeñas habitaciones sin ventanas.- Los fogones en los rincones sirven tanto como cocina y calefacción.

4. Recursos. - Los recursos naturales aprovechables para la economía nativa son algo diferentes en la costa y en las Sierra. Posiblemento las plantas silvestres y la vida animal fueron más abundantes en el remoto pasado, aunque la importancia de ambas pasó a sər secundaria cuando se incrementó la dependencia en las plantas y animales domesticados. Algunas plantas domesticadas son adaptables a todas las partes del país, tales como el maíz (en parte), los frijoles, las calabazas, los zapallos y el tabaco. Sin embargo, las habas, algunas especies de frijoles, maní, algódón y ají están restringidas a la costa y el tauwi, quinua, cañahua, papas, ocas, ollucos y mashua a la sierra. Tal vez el contraste mayor esté en la posibilidad de conseguir pescado y otros alimentos marinos en la costa, completando así la agricultura con la pesca, y la limitación de la llama a la altura, que permite así la ganadería como adición a la agricultura. Estos animales domésticos pueden ser criados en tierras no aptas para el cultivo y son importantes tanto por su lana como por la carne y también para el transporte.

Se puede lavar oro en la grava de las corrientes costeñas, pero los otros metales usados tan ampliamente en los tiempos precolombinos, cobre, oro, plata y estaño constituyen un recurso de la sierra. La costa tiene abundante arcilla para adobes y cerámica y la piedra aunque puede conseguirse se usa rara vez. Tanto la piedra como la arcilla son igualmente abundantes en la sierra. En realidad tal vez la piedra es 
demasiado abundante en lugares como Huari donde muchas de las paredes de piedra aparentemente fueron construídas a fin de limpiar de rocas la superficie. En ambas regiones los leños para construcciones o para combustible eran escasos. Esto era particularmente serio para la sierra donde el ichu, el estiércol de llama -takia- y la planta llareta ofrecían un combustible escasamente suficiente para la cociria pero rio para la calefacción.

Aunque existen muchos factores contrastantes entre la costa y la sierra que pueden haber afectado el desarrollo cultural, tres son probablemente los de mayor importancia. Primero, la necesidad de la adaptación biológica a la vida en la altura es de la mayor importancia, particularmente si éstas pueden mostrar a tipos físicos selectos. Hasta ahora los antropólogos físicos no han estudiado restos de esqueletos antiguos lo suficiente para determinar la existencia de tales diferencias, no obstante debe señalarse que Newman (1948) encontró por lo menos dos tipos raciales en la sierra, difiriendo ambos marcadamente de aquellos de la costa.

Segundo, son los rasgos que fomentan la uniformidad cultural en los valles de la costa comparados con aquellos que favorecen la variación cultural en las hoyas de la sierra. La unidad de algún tipo es casi una necesidad para las gentes agricultoras que habitan en valles aislados donde dependen de una sola fuente de agua para la irrigación. La falta de unidad es más probable en una hoya serrana desparramiada, donde cada sección puede ser económicamente independiente. La prehistoria de la costa presentan la evidencia de que los valles han sido controlados por pequeños grupos dominantes bajo un patrón de ciudades estado. En la sierra la unidad se alcanzó medjante confederaciones, o bien no se alcanzó.

Tercero, es el factor de la dificultad de vida en la altura en oposición a la costa. La vida en la sierra tiene una economía superflua, desde que todos sus aspectos requieren mayor energía para la misma recompensa. Por otro lado, la vida en los fértiles valles de la costa es relativamente fácil. Por lo menos los pueblos de la costa parecen haber disfrutado de mucho tiempo libre, sobre y bajo el mínimum de las actividades de subsistencia, en las que desarrollaron las artes y técnicas. El elaborado culto funerario, con su riqueza de ofrendas a los muertos es un fenómeno esencialmente costeño.

\section{TIPOS DE RELACIONES ENTRE LA COSTA Y LA SIERRA}

Esta breve revisión ha mostrado que existen muchos contrastes importantes entre la costa y la sierra que pudieron afectar el desarrollo cultural en las dos áreas. A pesar de esto, costa y sierra están claramente unidas cuando se considera la prehistoria del Perú en su totalidad. Es necesario por tanto examinar algunos de los posibles tipos de relación entre las dos áreas, y, donde la evidencia arqueológica lo permita, determinar cuales de estos son los más aplicables en diferentes tiempos y lugares. 
1.- Fondo cultural común.-- En tiempos antiguos el Perú puede haber contenido pueblos con diferentes patrones de subsistencia y culturales, pero a la vez se desarrolló la economía de agricultura intensiva, que se difundió en todas las partes del área y persistió hasta la conquista española, sino hasta la actualidad. Parece haber una pequeña duda acerca de si los muchos patrones regionales de subsistencia agrícola intensiva tuvieron un origen común. En realidad, muchos de los rasgos culturales que se encuentran en el Perú, tales como el putrón de los vestidos y el ceremonialismo religioso, no son específica. mente concomitantes con la agricultura intensiva. Así una cantidad de rasgos que unen las culturas de la costa con aquellas de la sierra pueden bien atribuirse a un origen común o a un desarrollo paralelo.

Cuando algunos ambientes peruanos se examinan desde el punto de vista de su adecuación para el cultivo intensivo, ciertos contrastes aparentes se minimizan. Por ejemplo, tanto los valles de la costa como las hoyas de la sierra tienen grandes áreas con tierras ricas, no erosionadas, adecuadas fuentes de agua, climas magníficos y con ausencia de foresta o cubiertos por hierba muy enraizada, difícil de erradicar con la simple técnica del palo de eavar.

2.- Difusión.-. En toda la prehistoria peruana, las grandes distancias fueron probablemente el factor que más afectó las comunicaciones. El viaje por tierra era esencialmente a pie, ya que la llama no es adecuada para para cabalgadura y porque la falta de materiales convenientes permitín sólo un desarrollo muy pequeño de los viajes por mar. Para el viaje a pie, los cerros y desiertos eran equivalentes de barreras y las distancias se median por el tiempo, no por millas. En consecuencia, la difusión directa entre las áreas habitables debe haber side mayor donde las distancias eran más cortas. Cuando se examina el Perú dosde este punto de vista se ve que las principales hoyas de la sierra estún ampliamente separadas unas de otras, mientras, que por otra parle, algunas no están tan distantes de sus vecinos valles costeños. En realidad, puede hacerse una nueva subdivisión del Perú que se base en las distancias antes que en las distinciones de costa y sierra (Bennett, 1948). Por ejemplo, una división del norte incluiría la hoya del Caileión de Huaylas y los valles costeños de Santa, Chimbote, Nepeña, Casma y tal vez Huarmey. Una división del centro tal vez combinaría la hoya del Mrntaro con los valles costeños desde Paramonga hasta Nazca. La división del norte es particularmente convincente para la arqueología, desde que el Callejón y los adyacentes valles costeños muestran ambos manifestaciones de. las culturas Chavín, Blanco sobre Rojo, Recuay, Tiahuanaco, y Negro-blanco-rojo. La división del centro Por lo menos el sitio de igual sentido cuando se sepa más del Mantaro. idénticos a aquellos de la costa.

Aunque estas ilustraciones sugieren que la costa y la sierra pueden no tener un contraste importante, cada división necesita ser examinada separadamente. Reichlen (1949), por ejemplo, no encuentra para Cajamarca relaciones con la costa hasta el periodo Tiahuanaco. Sin em- 
bargo, no es ilógico que los intercambios entre sierra y costa hayan sido favorecidos donde fuera posible, desde que los productos de cada región son bastantes diferentes.

3.- Migración.- Los movimientos verdaderos de pueblos deben constituir otro tipo de relación entre la costa y la sierra. Seguramente, la extensión de tales migraciones dependería de la magnitud del factor de aclimatación biológica y es interesante que todos los datos arqueológicos de las migraciones nos muestren movimientos de la sierra a la costa y no inversos. Esta prueba de las migraciones es grandemente inductiva. Para ilustración, cuando se encuentran estilos virtualmente idénticos de cerámica en un área de sierra y costa, tal como el estilo Recuay en el Callejón de Huaylas y en el bajo Santa, la migración está implícita. En Huaca Prieta, situada en el valle de Chicama, Bird (1948) encontró restos de una cultura que aparecía al fin de la secuencia precerámica. La nueva cultura produjo cerámica, maíz, calabaza averrugada, tejido verdadero y otras características. En este caso, es buena la evidencia de una migración, aunque no se conozca la fuente, si fué de la costa o de la sierra. Sin embargo, la cultura Gallinazo, debe haberse introducido al Valle de Virú por la región de la sierra, porque las secuencias conocidas en los adyacentes valles costeños no permiten suponer su desarrollo.

4.- Comercio.- Indudablemente el comercio fué un tipo importante de relación entre la costa y la sierra. La cerámica Mochica describe una gran variedad de plantas alimenticias, algunas de las cuales, tales como la papa, son variedades serranas que deben haberlas obtenido mediante el comercio. Los tejidos de Paracas hacen gran uso de lana de la sierra. El cobre que seiencuentra en numerosos implementos debe haber sido comerciado. Aparentemente la mayor parte de comercio era de materias primas antes que de artículos manufacturados. En Meso-América la interrelación de las áreas la determinan grandemente los objetos de comercio, pero en el Perú las piezas de comercio identificables son excesivamente raras.

5.- Peregrinaciones. - Los centros religiosos que atraían peregrinos de muchas partes del país se establecieron aparentemente en fecha muy antigua $\mathrm{y}$ han persistido hasta el presente. La prueba de algunos de estos ha sido presentadu en otra parte (Bennett y Bird, 1949), donde se consideraba como centros principales de peregrinaje a Chavín de Huántar, Tiahuanaco en Bolivia, Pachacamac en tiempo de la Conquista, y Copacabana en la actualidad. Tal patrón de peregrinaje sería un tipo importante de relación entre las áreas.

6.- Trabajo migratorio.- Hoy día, las migraciones de estación de trabajadores y sus familias de la sierra a la costa son todavía comunes. Grupos de trabajadores de los pueblos del Mantaro van a Cañete durante una estación todos los años. Tal viaje compromete a grupos de familias, no sólo a los trabajadores. Poco se conoce de la historia de es- 
te trabajo migratorio. Si el fenómeno fuera antiguo, bien puede haber constituído un factor de relación entre la costa y la sierra.

7.- Guerras y conquistas. - La guerra de regiones es en si misma un tipo de relación. La expansión Inca de la sierra hacia la costa está históricamente bien establecida como una conquista militar, y es probablemente cierto que la antigua expansión de Tiahuanaco fuera de la misma clase. El impacto de tales invasiones parece variado. La expansión de Tiahuanaco fué aparentemente motivada por un sentido religioso y su efecto en las culturas de la costa fué profundo. La conquista Inca fué política y en muchos lugares ocasionó únicamente cambios menores en las culturas locales según puede juzgarse por los hallazgos arqueológicos.

\section{LAS RELACIONES Y LOS PERIODOS DE TIEMPO}

Se han presentado diversos tipos de relaciones que han servido para unir las culturas de la costa con aquellas de la sierra. No hay razón lógica para que alguna o todas las combinaciones de este tipo puedan no haber estado operando al mismo tiempo. El factor de proximidad, como entre el Callejón de Huaylas y los valles de la costa favoreció la difusión, pero al mismo tiempo era apropiado para todos los otros tipos de relación. Sin embargo, un examen de los principales períodos de tiempo sugiere que ciertos tipos de relación fueron más activos en unos que en otros. Estos períodos, siguiendo las designaciones usadas por Bennett y Bird (1949) serán revisados, desde este aspecto.

1.- Primeros agriculores.ccilalliscasavevidencia sobre los antiguos cazadores, recolectores y pescadores del Perú no basta para hacer generalizaciones. Existen mayores datos para las poblaciones agrícolas precerámicas, aunque éstas estén limitadas a los valles de Chicama y Virú. Es dudoso que el Perú en su totalidad pueda en estos antiguos tiempos, ser llamado con propiedad una unidad cultural, y por consiguiente el problema de las relaciones entre la costa y la sierra es académico. Uno supondría que las diferencias ambientales tuvieron mayor efecto sobre las culturas que existieron antes de que la agricultura se convirtiera en la base principal de subsistencia.

2.- Los cultistas. - Este período marca el establecimiento de: un intensivo patrón agrícola de subsistencia, junto con una grande variedad de piantas domesticadas, cerámica, tejido, construcciones y otras manifestaciones de culturas bien logradas. Los restos arqueológicos de este período, identificados principalmente con el motivo estilizado de Chavín, se encuentran tanto en la costa como en la sierra. La gran similitud en la cerámica de sitios tan distantes como Chavín de Huántar y Ancón sugieren una migración y colonización. La arquitectura del sitio Chavín y el motivo felínico tan ampliamente difundidos sugieren igualmente que la peregrinación religiosa era un patrón importante. 
3.- Los experimentadores.- Este período se caracteriza por el desarrollo de las culturas locales, y por experimentos en todos los aspectos tecnológicos. Las evidencias comparativas muestran que una técnica descubierta en un área, tal como la pintura en la cerámica de tipo positivo blanco sobre rojo o negativa resistente, se extendía rápidamente en otras áreas. Posiblemente tal expansión se realizaba mediante la difusión, desde que no hay pruebas de migraciones o de peregrinaciones religiosas.

En este tiempo, las culturas de la costa usaban tanto el cobre como la lana, implicándose así el establecimiento del comercio de materias primas.

4.- Los maestros artesanos.- El desarrollo de las técnicas y la perfección de la artesanía trajo como resultado una intensificación de las culturas regionales, tales como la Mochica, Nazca, Recuay y Pucará. El comercio fué ciertamente importante, aunque básicamente en materias primas. Ciertos motivos ampliamente extendidos, tales como el pezrayo, el felino, la figura corredora, pueden indicar difusión, aunque sus expresiones locales sugieren más la herencia de un fondo cultural común. Las guerras locales fueron evidentemente importantes y la expansión en la forma de conquista fué tomando lugar en la costa y la única indicación que esto implica tanto a la costa como a la sierra es la expansión de Recuay en el callejón de Huaylas hacia el bajo Santa.

5.- Los expansionistas.- La gran expansión de Tiahuanaco sobre casi todo el Perú fué indudablemente por conquista, sin considerar si fué motivada política o religiosamente. En todas partes Tiahuanaco se combina con los estilos docales como elemento dominante o los reemplaza totalmente. El patrón de peregrinación fué también importante otra vez. Es interesante que los datos físicos muestren que los tipos raciales serranos aparezcan en la costa durante este período.

6.- Constructores de ciudades.- Siguiendo a la unidad pan-peruviana de la expansión Tiahuanaco, las culturas regionales florecieron nuevamente, aunque con el incremento del conocimiento de sus vecinos. La guerra era importante e intentaban la expansión local y la conquista. La migración y la colonización se indican por la expansión de Chimú hacia el norte y sur y por la expansión de Cajamarca hacia la costa. La variedad de materiales hallados en todas las culturas regionales muestran que el comercio era activo.

7.- Los imperialistas. - La expansión Inca fué una conquista política seguida por la incorporación de los pueblos conquistados dentro de un sistema imperial. El comercio, la colonización (mitimaes) y otros tipos de relación fueron muy importantes aunque ejercidos bajo controles estatales. 


\section{CONCLUSIONES}

Esta revisión de las relaciones entre la costa y la sierra muestra varios caminos, mediante los cuales la prehistoria peruana pudo haber estado unida a pesar de sus subdivisiones características. Sugiere también que los grandes contrastes ambientales de la costa y la sierra no fueron siempre una causa de bifurcación cultural. Más bien, que la unidad o falta de unidad cultural depende más del tipo de relaciones que de las diferencias ambientales. Todo este problema de las relaciones evidentemente implica algo más que el puro estudio arqueológico. El período histórico introduce nuevas economías, nuevos controles políticos, nuevas técnicas y en general cambios fundamentales para el $\mathrm{Pe}$ rú. Aunque la magnitud de estos cambios parezcan separar su estudio de lo prehistórico, en realidad podría hacerse mucho de interés mutuo. ¿Qué condiciones históricas efectuaron los cambios más profundos en las culturas indígenas? ¿Qué significa un nuevo sistema de transporte, una nueva vía férrea o una carretera, para la relación actual de la costa y la sierra? Las conclusiones acertadas deben aguardar la colaboración de todos los estudiosos del Peru, contemporáneos y del pasado.

\section{B I B LI O G R A I A}

BENNETT, Wendell C.- 1948. The Peruvian Co - Tradition (Memoirs of the Society for American, № 4, pp. 1-7, Menasha, 1948).

1950. Cultura! Unity and Disunity in the Titicaca Basin (American Antiquity, vol. 16. No 2, Menasha, 1950).

BENNETT, Wendell $C$ and Junius Bird. 1949. Andean Culture History, New York, 1949). Orge Puccinelli ConversO"

BIRD, Junius - 1948. Proceramic Cultures in Chicama and Virú (Memoirs of the Society for American Archaelogy, № 4, pp. $21-28$. Menasha).

MEANS. Philip A. - 1931. Ancient Civilizations of the Andes, (New York, 1931).

MONGE. Carlos.- 1948. Acclimatization in the High Andes, (Baltimore, 1948).

NEWMAN, Marshall T.- 1948. - A Summary of the Racial History of the Peruvian Area, Memoire of the Society for American Archeology, No 4, pp. 16-19, Menasha, 1948).

REICHLEN, Henry et Paule- - 1949.- Recherches Archcologiques dans les Andes de Cajamarca, (Journal de la Societé des Americanistes, ns, vol. 38, pp. $137-174$, Paris, 1949).

ROWE, John Howlnad.- 1948. On Basic Highland Cultures, (Memoirs of the Society for American Archaeology, No 4, p. 20, Menasha, 1948). 